\title{
Continuous four-channel EEG monitoring in the evaluation of echodense ultrasound lesions and cystic leucomalacia
}

\author{
J CONNELL, R OOZEER, R REGEV, L S DE VRIES, L M S DUBOWITZ, AND \\ $\mathrm{V}$ DUBOWITZ
}

Department of Paediatrics and Neonatal Medicine, Royal Postgraduate Medical School, Hammersmith Hospital, London

SUMMARY Continuous monitoring by electroencephalography (EEG) was performed in 31 preterm infants with echodense ultrasound lesions using the Oxford Medilog four channel recorder. In 12 infants these densities preceded cyst formation, eight in the periventricular and four in the subcortical region. In the 19 other infants the densities resolved.

The most severe EEG depression occurred in the four infants with subcortical cysts, who also had the poorest outcome. Six of eight infants with periventricular lesions had lesser degrees of abnormality though all infants had moderate to severe handicap at follow up. Similar abnormalities were seen on EEG recording in three infants in whom the densities resolved without cyst formation and these infants also had an abnormal outcome.

An abnormal EEG recording provides a sensitive early guide to the severity and prognosis of these lesions, even before they can be seen on ultrasonography.

Recent refinements in ultrasound techniques have permitted diagnosis of ischaemic as well as haemorrhagic lesions. ${ }^{12}$ Ischaemic lesions initially present as areas of increased echodensity on ultrasonography; some resolve but others proceed to extensive cyst formation in the periventricular or subcortical white matter, referred to as periventricular or subcortical leucomalacia. The poor prognosis of infants with extensive cystic lesions has been well documented, but in those whose densities resolve the prognosis is more variable. ${ }^{3-5}$

Although prognosis based on the presence of extensive cysts seems to be reliable, the cysts are not present initially and may take two to four weeks to develop. Further aids to early prognosis would therefore be valuable. This study aimed to assess the prognostic value of early and sequential EEG recording in infants with echodense ultrasound lesions and subsequent extensive cystic leucomalacia.

\section{Method}

Over 21 months 150 infants were entered into a prospective correlative study of EEG recordings and cranial ultrasonography. The 31 infants with persistent echodensities are reported here. Their mean gestation was 31 weeks (range 27-36) and mean birth weight $1230 \mathrm{~g}$ (range 720-2150).

\section{ULTRASOUND ASSESSMENT}

Ultrasound assessment was made daily during the first week of life and then twice weekly, using an ATL Mark 3 sector scanner (3.5-7.5 MHz crystal). Densities were defined as unilateral or bilateral areas of increased echogenicity seen in both coronal and parasagittal views that persisted for at least 48 hours. Their evolution was classified as resolution without cyst formation, or extensive cyst formation in the periventricular or subcortical white matter.

\section{EEG ASSESSMENT}

An Oxford Medilog 4-24 continuous four channel monitor was used. ${ }^{6}$ Electrocardiogram, respiration, and two channels of EEG were recorded with silver/silver chloride electrodes applied with collodion in the F4-P4, F3-P3 positions (impedances $<5$ kohms).

Initial recordings were made within 48 hours of birth, transfer to the neonatal unit, or onset of the 
illness (if delayed). Sequential recordings were made for 24 hour periods at intervals of two to four weeks until term or discharge from the unit. Data for each 24 hour period were recorded on standard C 120 cassette tape and reviewed on a visual display unit of Medilog system at 20 and 60 times the normal speed at scales of 1.5 and $3.0 \mathrm{~cm} /$ second.

Compressed transcription was done at $6 \mathrm{~cm} /$ minute using a Siemens 8 Elema Schonander ink jet recorder. All initial recordings were assessed independently by two observers without knowledge of the ultrasound development or clinical outcome.

Classification of normal or abnormal was made from standards for gestational age established for the Medilog 4-24 monitor in a group of low risk preterm infants, ${ }^{7}$ as well as from criteria derived from conventional EEG recordings. ${ }^{8}$

EEG abnormalities were classified into four categories (fig 1), as follows:

(1) Activity inappropriate for gestation. In preterm infants this was indicated by excess discontinuous activity or a prolonged interburst interval for
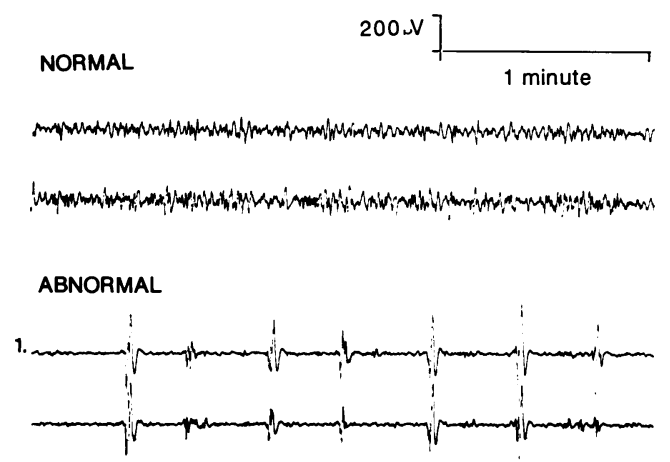

2.
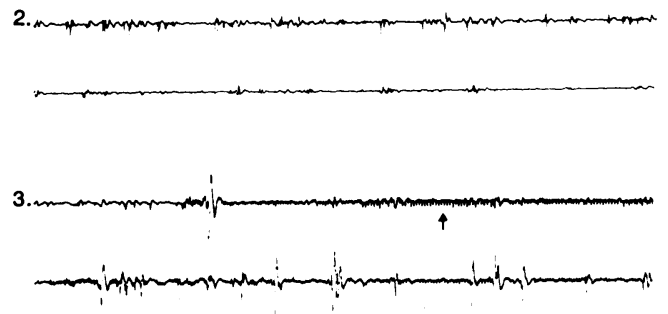

Fig 1 Examples of categories of EEG abnormality (compressed time scale) preceded for contrast by normal preterm record during phase of continuous activity. gestational age, and in term infants by impaired sleep state definition.

(2) Overtly abnormal activity independent of gestation (excluding seizures) as indicated by: persistent discontinuity throughout recording or burst suppression; low amplitude recording; asymmetry; asynchrony; and spikes and sharp waves.

These were further classified according to background activity into those showing: (i) one abnormal feature on normal background; (ii) those showing one abnormal feature on category 1 abnormal background; and (iii) those showing two or more overtly abnormal features.

(3) Seizures were further classified according to background activity into; (i) those on normal background; (ii) those on category 1 abnormal background; and (iii) those on category 2 abnormal background.

(4) Maximal background depression either (i) with seizures or with (ii) no discernible activity.

NEUROLOGICAL ASSESSMENT OF OUTCOME

Assessment of outcome was made on clinical examination at discharge from the neonatal unit using a standardised protocol, ${ }^{9}$ and at 6-18 months using the Griffiths mental development scale and items from Amiel-Tison's ${ }^{10}$ and Touwen's ${ }^{11}$ scores.

Because some infants have been assessed only up to the age of 6 months, only major neurological abnormalities were considered in this study. These included confirmed cerebral palsy and mental retardation.

\section{Results}

The table summarises the development of the ultrasound appearances, the initial and sequential EEG findings, and the clinical outcome in the 31 infants studied. The relative timings of the development of the EEG and ultrasonographic abnormalities are also shown.

\section{ULTRASOUND ASSESSMENT}

In 19 of the infants studied the densities resolved without cyst formation, but in seven they proceeded to extensive cystic leucomalacia. In five infants the cysts were already well established at presentation. Of the 12 infants with cysts, eight had periventricular leucomalacia and four had subcortical leucomalacia.

EEG ASSESSMENT

In 13 infants the initial EEG recording was abnormal (fig 2). Category 4 abnormalities were found in four infants, two of whom presented with subcortical leucomalacia and two of whom subsequently 
Table Summary of ultrasound development, initial and sequential EEG findings, and outcome in 31 infants with echodense lesions on ultrasonography

\begin{tabular}{|c|c|c|c|c|}
\hline $\begin{array}{l}\text { Case } \\
\text { No }\end{array}$ & Ultrasound development & $\begin{array}{l}\text { Initial } E E G \\
\text { category }\end{array}$ & $\begin{array}{l}\text { Sequential EEG } \\
\text { category }\end{array}$ & Outcome \\
\hline 1 & Cysts: subcortical leucomalacia & 4 (ii) & Not done & Died \\
\hline 2 & Cysts: subcortical leucomalacia & 4 (ii) & Not done & Died \\
\hline 3 & Cysts: subcortical leucomalacia** & 4 (i) & 3 (iii) & Abnormal \\
\hline 4 & Cysts: subcortical leucomalacia** & 4 (i) & 3 (iii) & Abnormal \\
\hline 5 & Cysts: periventricular leucomalacia* & 3 (iii) & Not done & Died \\
\hline 6 & Cysts: periventricular leucomalacia* & 3 (iii) & 2 (ii) & Died \\
\hline 7 & Cysts: periventricular leucomalacia & 3 (iii) & 2 (ii) & Abnormal \\
\hline 8 & Cysts: periventricular leucomalacia & 2 (iii) & Not done & Abnormal \\
\hline 9 & Cysts: periventricular leucomalacia* & 2 (ii) & Normal & Abnormal \\
\hline 10 & Cysts: periventricular lcucomalacia** & 1 & 3 (i) & Died \\
\hline 11 & Cysts: periventricular Icucomalacia & Normal & Not done & Abnormal \\
\hline 12 & Cysts: periventricular leucomalacia & Normal & Normal & Abnormal \\
\hline 13 & Densities only & 3 (iii) & Not done & Died \\
\hline 14 & Densities only & 3 (iii) & 3 (i) & Abnormal \\
\hline 15 & Densities only & 2 (iii) & Not done & Abnormal \\
\hline 16 & Densities only & Normal & Not done & Normal \\
\hline 17 & Densities only & Normal & Not done & Normal \\
\hline 18 & Densities only & Normal & Not done & Normal \\
\hline 19 & Densities only & Normal & Not done & Normal \\
\hline 20 & Densities only & Normal & Not done & Normal \\
\hline 21 & Densities only & Normal & Not done & Normal \\
\hline 22 & Densities only & Normal & Not done & Normal \\
\hline 23 & Densities only & Normal & Not done & Normal \\
\hline 24 & Densities only & Normal & Normal & Died \\
\hline 25 & Densities only & Normal & Normal & Normal \\
\hline 26 & Densities only & Normal & Normal & Normal \\
\hline 27 & Densities only & Normal & Normal & Normal \\
\hline 28 & Densities only & Normal & Normal & Normal \\
\hline 29 & Densities only & Normal & Normal & Normal \\
\hline 30 & Densities only & Normal & Normal & Normal \\
\hline 31 & Densities only & Normal & Normal & Normal \\
\hline
\end{tabular}

${ }^{*}$ EEG abnormality preceded cysts; **EEG abnormality preceded densities.

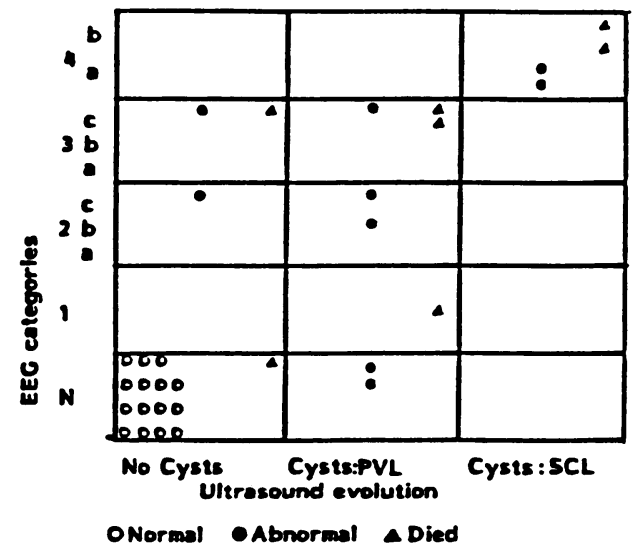

Fig 2 Correlation of initial EEG, ultrasound evolution of densities, and neurological outcome in 31 infants.

developed it. Eight infants had category 2 and 3 abnormalities, five of which were associated with cystic lesions. Two of these had presented with periventricular leucomalacia and another three sub- sequently developed it. The remaining three infants with category 2 and 3 abnormalities did not develop cysts. Only one infant had category 1 abnormalities and he subsequently developed periventricular leucomalacia. The EEG abnormality preceded the appearance of even the density in three of the above infants; two of these subsequently developed subcortical leucomalacia and one periventricular leucomalacia. Fig 3 shows the progression in one of these infants.

Eighteen infants had normal EEG recordings. Of these 16 did not form cysts; one had presented with periventricular leucomalacia and one subsequently developed it.

Six infants had persistently abnormal EEG recordings (fig 4), including two with subcortical leucomalacia, three with periventricular leucomalacia, and one without cysts. Ten infants had normal sequential EEG recordings. In one infant with periventricular leucomalacia an initially abnormal recording became normal within two weeks. Nine infants had persistently normal EEG recordings, 

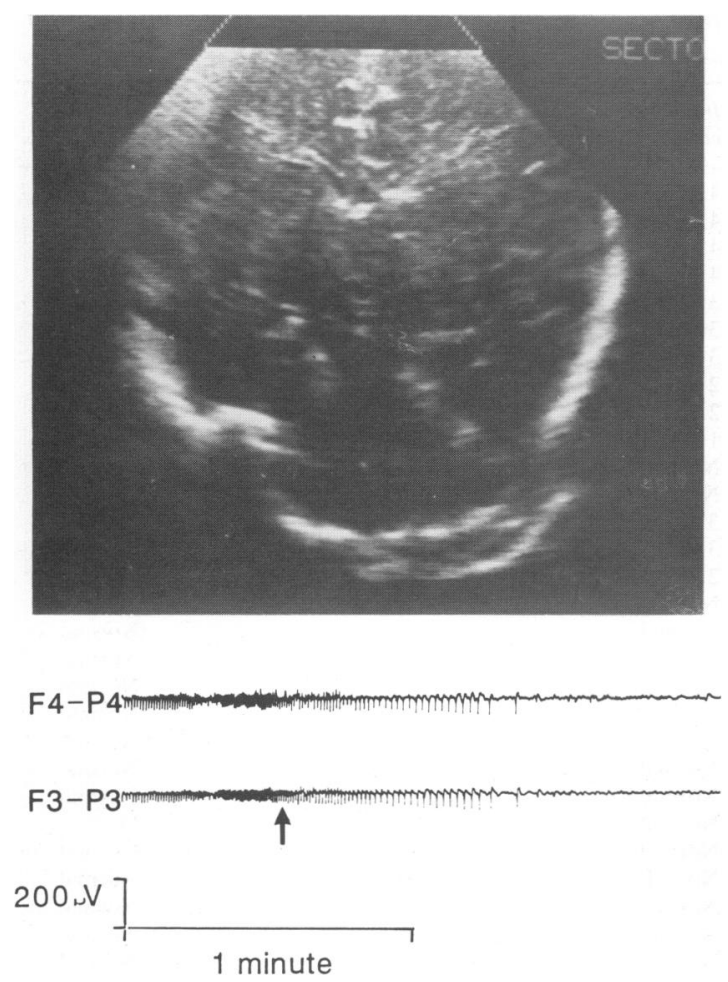

Fig 3(a) Relative evolution of EEG and ultrasound abnormalities of subcortical leucomalacia in 35 week gestation infant with necrotising enterocolitis (case 3 ). Day 1, ultrasound within normal limits; EEG severely abnormal with bilateral seizures (arrowed) on very low amplitude background.

including eight without cysts and one with periventricular leucomalacia.

Eight infants showed seizure activity on EEG recording (categories 3 and 4), including two with subcortical leucomalacia, four with periventricular leucomalacia, and two without cysts. These were associated with clinical seizures in five infants.

NEUROLOGICAL OUTCOME

Eight infants died too early for assessment and nine of the 23 survivors developed abnormally.

CORRELATION OF EEG WITH CLINICAL OUTCOME

The two survivors who showed category 4 abnormalities on the initial EEG recordings had the most severe neurological abnormalities (fig 2). Category 2 and 3 abnormalities were associated with abnormal outcome in seven surviving infants. Category 1 abnormality was found in only one infant who died.

A normal initial EEG recording was not invari- ably associated with a normal outcome; two survivors had normal initial recordings but abnormal outcome.

If the sequential EEG recordings were persistently abnormal the outcome was always abnormal; four survivors in whom this occurred were all neurologically abnormal. Not all infants with a normal sequential EEG had a normal outcome, however. Neither persistently normal recordings nor ones which subsequently became normal were constantly associated with normal outcome. This happened in seven survivors, in two of whom the outcome was abnormal.

Seizures, present in four infants, always resulted in abnormal outcome.

\section{Discussion}

EEG recordings in neonates have been shown in other conditions ${ }^{1213}$ to give early and sensitive

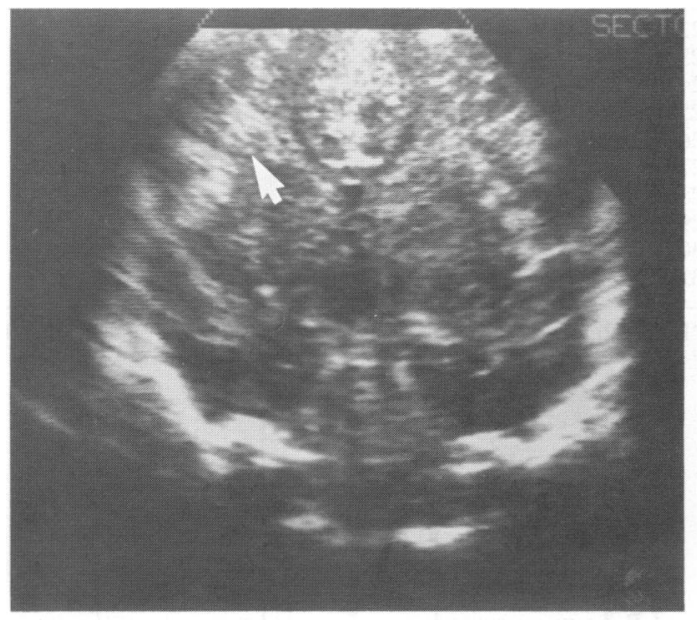

F4-P4

F3-P3

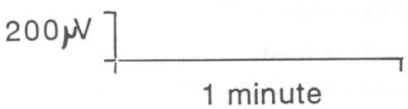

Fig 3(b) Relative evolution of EEG and ultrasound abnormalities of subcortical leucomalacia in 35 week gestation infant with necrotising entercolitis (case 3).

Day 5, ultrasound now abnormal with echodensities in periventricular regions and inter-hemispheric fissure; $E E G$ persistently abnormal with extreme amplitude depression. 


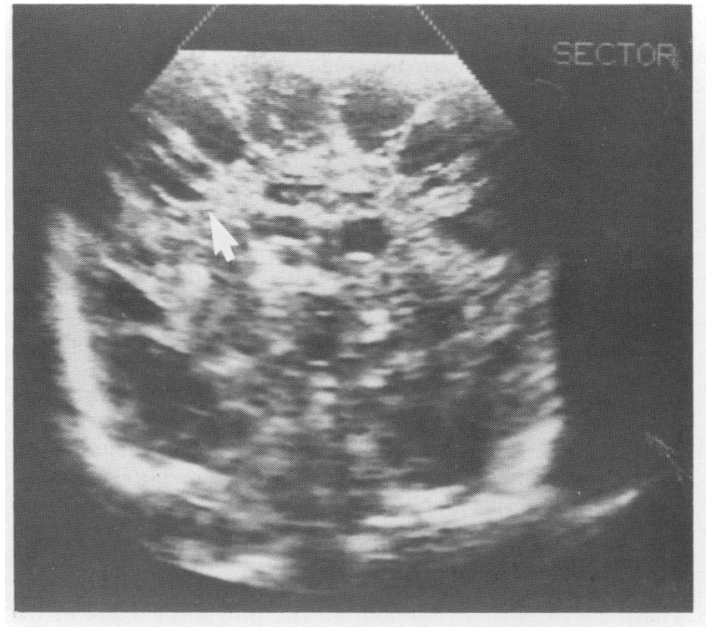

F4-P4
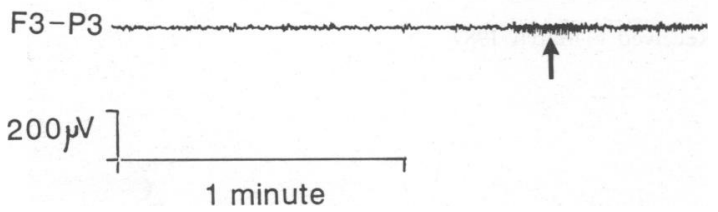

Fig 3(c) Relative evolution of EEG and ultrasound abnormalities of subcortical leucomalacia in 35 week gestation infant with necrotising entercolitis (case 3). Week five; ultrasound now shows extensive subcortical leucomalacia; EEG still low amplitude and discontinuous with persistent seizures (arrowed).

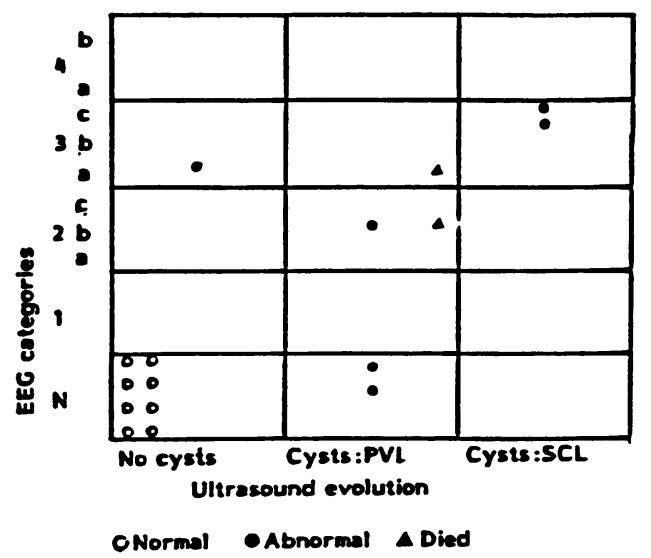

Fig 4 Correlation of sequential EEG, ultrasound evolution of densities, and neurological outcome in 16 infants. indications of the functional severity of lesions of considerable prognostic importance. This study shows that the technique may also be applied to the recently recognised problem of whether ultrasound echodensities indicate cystic leucomalacia.

In this condition the EEG recording also provided a sensitive guide to the functional severity of the ultrasonographic lesion, showing much more frequent and serious abnormalities in infants with cystic leucomalacia than in those whose densities resolved without cysts. In particular, it identified the exceptional severity of the functional damage associated with subcortical leucomalacia. Maximal depression of background EEG activity was seen in the four infants with subcortical leucomalacia but in no other group.

The sensitivity of the initial EEG abnormality as an index of the functional severity of the lesion was also reflected in its prognostic ability. Although there was a definite correlation between EEG abnormalities and abnormal outcome, a normal EEG recording was not invariably associated with a normal clinical outcome. It is possible that with only two channels of EEG recording some abnormal foci may have been missed. Alternatively, the EEG recording may have shown some recovery after the initial insult. In favour of this argument is the fact that improvement or return to normal of an initially abnormal EEG recording was seen in seven infants in sequential studies. Two infants had normal initial recordings and abnormal outcome. One of these was studied late when cysts were already present. The other had densities from day 1 after a pregnancy complicated by severe pre-eclamptic toxaemia. It is therefore possible that the EEG, which was also monitored from day 1 , may already have become normal by the time of the birth after an insult in utero.

In contrast, the particular prognostic value of an abnormal EEG recording is well illustrated by this study. Seizures always indicated an abnormal outcome. Even in the absence of seizures, however, abnormalities such as low amplitude and excessive discontinuous background activity, and asymmetry and asynchrony of discharges, were consistently adverse prognostic factors, irrespective of ultrasonographic findings.

The particular additional value of EEG monitoring was that the prognostically relevant information that it supplied was immediately available from the first day of recording, whereas that derived from ultrasonography was not available for two to four weeks when the densities had resolved or cysts had formed.

Neonatal EEG recordings may be effectively monitored using the four channel Medilog 4-24 


\section{Connell, Oozeer, Regev, de Vries, Dubowitz, and Dubowitz}

continuous recorder in infants who have ultrasound echodensities and cystic leucomalacia; the information that the recordings provide acts as a sensitive index of the severity of such lesions. The early appearance of these important EEG changes means that the role of this investigation is not confined merely to confirming the ultrasound assessment. Rather it provides the first available guide to assessing the functional severity of these lesions and their prognostic importance.

\section{References}

' Hill A, Melson GL, Clark HB. Hemorrhagic periventricular leukomalacia: diagnosis by real-time ultrasound and correlation with autopsy findings. Pediatrics 1982;69:282-4.

${ }^{2}$ Levene MI, Wigglesworth JS, Dubowitz V. Hemorrhagic periventricular leukomalacia in the neonate: a real-time ultrasound study. Pediatrics 1983;71:794-7.

3 McMenamin JB, Shackleford GD, Volpe JJ. Outcome of neonatal IVH with periventricular echodense lesions. Ann Neurol 1984;15:285-90.

${ }^{4}$ Weindling AM, Rochefort MJ, Calvert SA, Folk TF, Williamson A. Development of cerebral palsy after sonographic detection of periventricular cysts in the newborn. Dev Med Child Neurol 1985;27:800-6.

5 Fawer C-L, Calane A, Farrer M-T. Neurodevelopmental outcome at 12 months of age related to cerebral ultrasound appearances of high-risk preterm infants. Early Hum Dev $1985 ; 2: 123-32$.

${ }^{6}$ Eyre JA, Oozeer RC, Wilkinson AR. Diagnosis of neonatal seizure by continuous recording and rapid analysis of the electroencephalogram. Arch Dis Child 1983;58:785-90.

7 Connell JA, Oozeer RC, Dubowitz V. Continuous 4-channel EEG monitoring: a guide to interpretation, with normal values, in preterm infants. Paediatrics (in press).

${ }^{8}$ Werner SS, Stockard JE, Bickford RG. Atlas of neonatal electroencephalography. New York: Raven Press, 1977.

9 Dubowitz LMS, Dubowitz V. Neurological assessment of the preterm and full-term newborn infant. Clinics in developmental medicine, no 79. Oxford: Blackwell Scientific Publications, 1981.

10 Amiel-Tison C, Grenier A. Evaluation neurologique du nouveau-ne et du nourisson. Paris: Masson, 1980.

11 Touwen BLC. Examination of the child with minor neurological dysfunction. Clinics in developmental medicine, no 71. Oxford: Blackwell Scientific Publications, 1979.

12 Monod N, Pajot N, Guidasci S. The neonatal EEG: statistical studies and prognostic value in fullterm and preterm babies. Electroencephalogr Clin Neurophysiol 1972;32:529-44.

13 Tharp BR, Cukier F, Monod N. The prognostic value of the EEG in premature infants. Electroencephalogr Clin Neurophysiol 1981;51:219-36.

Correspondence to Dr V Dubowitz, Department of Paediatrics and Neonatal Medicine, Royal Postgraduate Medical School, Hammersmith Hospital, London W12 0JS.

Received 6 March 1987 